\title{
Cell-Mediated Immune Responses to Complex and Single Mycobacterial Antigens in Tuberculosis Patients with Diabetes
}

\author{
Abu S. Mustafa ${ }^{a}$ Abdulsalam M. El-Shamy ${ }^{b}$ Nada M. Madi ${ }^{a}$ \\ Hanady A. Amoudy ${ }^{a}$ Rajaa Al-Attiyah ${ }^{a}$ \\ ${ }^{a}$ Department of Microbiology, Faculty of Medicine, Kuwait University, and \\ ${ }^{b}$ Chest Diseases Hospital and Allergy Centre, Ministry of Public Health, Kuwait
}

\author{
Key Words \\ Diabetes - Tuberculosis - Mycobacterium tuberculosis • \\ Antigens $\cdot$ Cellular immune responses
}

\begin{abstract}
Objective: To evaluate cell-mediated immune (CMI) response in diabetic and non-diabetic tuberculosis (TB) patients and healthy subjects in response to complex, fractionated and single antigens of Mycobacterium tuberculosis. Material and Methods: Peripheral blood mononuclear cells (PBMC) were obtained from patients suffering from pulmonary TB and type II diabetes $(n=7)$, pulmonary TB without diabetes $(n=10)$ and healthy subjects without TB and diabetes $(n=10)$. PBMC were assessed for CMI responses in antigen-induced proliferation assays in response to complex mycobacterial antigens (whole cells, cell walls and culture filtrate of $M$. tuberculosis), a battery of naturally purified or recombinant produced secreted (ESAT6, MPT59, MPT64 and MTB38) and cytosolic (MTB10, MTB70, ML10, ML28, ML36, ML65 and MB65) mycobacterial antigens and fractionated culture filtrate proteins (fractions F1-F10) of M. tuberculosis. Results: The majority ( $>70 \%$ ) of diabetic and non-diabetic TB patients and healthy subjects responded to the complex antigens of $M$. tuberculosis. However, among the single antigens, ESAT6 was most frequently recognized by TB patients with and without diabetes, but least recognized by healthy subjects. The secreted antigens MPT59 and MPT64 were rec-
\end{abstract}

ognized by all the groups, whereas the cytosolic antigens were recognized best by healthy subjects. When tested with fractionated secreted proteins present in the culture filtrate of $M$. tuberculosis, the best responses in both diabetic and non-diabetic TB patients were obtained with fractions containing low-molecular-weight proteins. Conclusions: Diabetic and non-diabetic TB patients respond frequently to secreted low-molecular-weight ESAT6 antigen of $M$. tuberculosis, indicating that this antigen may be useful in the diagnosis of TB in both the groups.

Copyright $\odot 2008$ S. Karger AG, Base

\section{Introduction}

Tuberculosis (TB) and diabetes are among the most important public health problems worldwide. TB ranks among the top 10 causes of global mortality $[1,2]$. It is estimated that approximately one third of the world's population is infected with Mycobacterium tuberculosis; 8 million people develop active TB leading to about 2 million deaths each year [2]. Furthermore, TB costs more than USD 3.3 billion a year in lost productivity [3]. With respect to diabetes, the statistics released by the World Health Organization and the International Diabetes Federation are alarming [4]. The number of diabetics in the world is expected to increase from 194 million in 2003 to 330 million in 2030 , with $75 \%$ diabetic patients living in

\section{KARGER}

Fax +4161306 1234

E-Mail karger@karger.ch

www.karger.com
(C) 2008 S. Karger AG, Basel

$1011-7571 / 08 / 0174-0325 \$ 24.50 / 0$

Accessible online at:

www.karger.com/mpp
Prof. Abu Salim Mustafa

Department of Microbiology, Faculty of Medicine, Kuwait University PO Box 24923

13110 Safat (Kuwait)

Tel. +965 4986 505, Fax +965 5332 719, E-Mail abusalim@hsc.edu.kw 
developing countries [4]. In some countries of the Middle East, 1 in 4 deaths in adults aged between 35 and 64 years is attributable to diabetes [4]. According to the National Institute of Diabetes and Digestive Kidney Disease and the American Diabetes Association, diabetes was the sixth leading cause of death in 1999 with a direct cost of USD 44 billion and an indirect cost of USD 54 billion annually. In 2002, the direct and indirect cost totaled USD 132 billion [4].

In addition to being two independent major health problems of infectious and non-infectious nature, an association has been observed [5] between TB and diabetes. Incidence of $\mathrm{TB}$ among diabetic patients is $2-5$ times higher when compared to the general population [5]. Before the discovery of insulin, a diagnosis of diabetes was a death sentence within 5 years, and the usual cause of that death was TB [5]. In general, diabetes is easy to detect, whereas the diagnosis of TB poses a challenge. The study of cell-mediated immune (CMI) responses against the antigens of $M$. tuberculosis is important to identify antigens of diagnostic relevance [6-10]. In order to identify antigens that could be of diagnostic relevance in diabetic TB patients, this study was conducted to evaluate CMI responses to complex and single secreted and cytosolic antigens of $M$. tuberculosis in diabetic and non-diabetic TB patient as well as in healthy subjects without TB and diabetes.

\section{Materials and Methods}

Patients and Isolation of Peripheral Blood Mononuclear Cells

Heparinized blood was collected from clinically, radiologically and bacteriologically confirmed cases of pulmonary TB attending the Chest Diseases Hospital, Kuwait and suffering from type II diabetes mellitus $(n=7)$ and without diabetes $(n=10)$. In addition, blood was also obtained from 10 healthy subjects without TB and diabetes donating blood at the Central Blood Bank, Kuwait. All of the patients and healthy subjects were HIV-negative. Collection of blood samples and the study was approved by the Ethics Committee, Faculty of Medicine, Kuwait University, Kuwait.

Peripheral blood mononuclear cells (PBMC) were isolated from the heparinized blood of patients and control according to standard procedures [11]. In brief, the blood from each individual was diluted with warm tissue culture medium (RPMI-1640, Gibco-BRL) at a ratio of 1:2 and gently mixed. Two volumes of the diluted blood were loaded on top of 1 volume of a Lymphoprep gradient (Pharmacia Biotech, Uppsala, Sweden). After centrifugation, the white ring of PBMC between the plasma and the Lymphoprep was removed and washed 3 times with RPMI-1640. The cells were finally suspended in complete tissue culture medium [RPMI-1640 + 10\% human AB serum + penicillin $(100 \mathrm{U} / \mathrm{ml})+$ streptomycin $(100 \mu \mathrm{g} / \mathrm{ml})+$ gentamycin $(40 \mu \mathrm{g} / \mathrm{ml})+$ fungizone
$(2.5 \mu \mathrm{g} / \mathrm{ml})]$ and counted with a coulter counter (Coulter Electronics Ltd., Luton, Beds., England).

\section{Complex and Fractionated Mycobacterial Antigens}

The complex antigens were killed whole cells of $M$. tuberculosis H37Ra [11], M. tuberculosis culture filtrate (MT-CF) highly enriched for secreted antigens and purified M. tuberculosis cell walls (MT-CW) [12]. MT-CF and MT-CW were kindly provided by Dr. P.J. Brennan (Colorado State University, Fort Collins, Colo., USA) through the repository of TB research materials, NIH contract No. AI-25147, USA. The fractionated culture filtrate protein antigens ranging from low- to high-molecular-weight fractions (F1-F10, provided by Dr. P. Andersen, Statens Serum Institute, Copenhagen, Denmark) were prepared by using preparative sodium dodecyl sulfate-polyacrylamide gel electrophoresis (SDSPAGE) according to methods described previously [13]. All fractions were stabilized with $2 \%$ human $\mathrm{AB}$ serum and stored frozen at $-20^{\circ} \mathrm{C}$ until use.

\section{Purified Mycobacterial Antigens}

The recombinant ESAT6 antigen (provided by Dr. P. Andersen, Statens Serum Institute) was expressed in Escherichia coli and purified, as previously described [14]. The other recombinant mycobacterial antigens MTB10, ML28, ML65, ML10, ML36, MTB38, MTB70 and MB65 and the control antigen maltose binding protein (MBP) were provided by G. Singh (Gene Expression, GBF, Braunschweig, Germany) with financial support from the Immunology of Mycobacteria (IMMYC) component of the United Nations Development Program (UNDP)/World Bank/WHO Special Program for Research and Training in Tropical Diseases. Among the above antigens, ESAT6 and MTB38 are secreted whereas others belong to the group of cytosolic mycobacterial antigens [14].

Highly purified secreted antigens MPT59 (lot 12455A2) and MPT64 (lot 6523A2) were prepared from the mycobacterial culture filtrates as previously described [14]. The same lot of each antigen (provided by Dr. H.G. Wiker, Division for Environmental Medicine, Norwegian Institute of Public Health, Oslo, Norway) was used throughout the study.

\section{Antigen-Induced Proliferation of PBMC}

Antigen-induced proliferation of PBMC was performed by using standard procedures $[15,16]$. In brief, PBMC $\left(2 \times 10^{5}\right.$ cells/ well) suspended in $50 \mu \mathrm{l}$ of complete tissue culture medium were seeded into the wells of 96 -well tissue culture plates (Nunc, Roskilde, Denmark). Antigen in $50 \mu \mathrm{l}$ of complete medium was added to the wells in triplicate at an optimal concentration of $5 \mu \mathrm{g} / \mathrm{ml}$. Whole-cell M. tuberculosis was used at a concentration of $10 \mu \mathrm{g}$ (wet weight) $/ \mathrm{ml}$. The final volume of the culture in each well was adjusted to $200 \mu$ l. The plates were incubated at $37^{\circ} \mathrm{C}$ in a humidified atmosphere of $5 \% \mathrm{CO}_{2}$ and $95 \%$ air. The cultures were pulsed on day 6 with $1 \mu \mathrm{Ci}$ of $\left[{ }^{3} \mathrm{H}\right]$-thymidine (Amersham Life Sciences, Little Chalfont, UK) for $4 \mathrm{~h}$, harvested on filter mats with a Skatron harvester (Skatron Instruments AS, Oslo, Norway), and the radioactivity incorporated was measured by liquid scintillation counting. The radioactivity incorporated was obtained as counts per minute (cpm). The average cpm was calculated from triplicate cultures stimulated with each antigen as well as from triplicate wells of negative control cultures lacking antigen. Cellular proliferation results were used to calculate stimulation index (SI), which is de- 
Table 1. Antigen-induced proliferation responses of PBMC from diabetic pulmonary TB patients in response to complex and single secreted and cytosolic antigens of M. tuberculosis

\begin{tabular}{|c|c|c|c|c|c|c|c|c|}
\hline Antigen & \multicolumn{7}{|c|}{ Antigen-induced proliferation (SI) of PBMC from patients } & $\mathrm{P} / \mathrm{T}$ \\
\hline M. tuberculosis & 0.7 & 1.8 & 182 & 234 & 59 & 579 & 630 & $5 / 7$ \\
\hline MT-CF & 1.0 & 3.5 & 180 & 58 & 2.7 & 632 & 726 & $6 / 7$ \\
\hline MT-CW & 2.2 & 10.2 & $\mathrm{ND}$ & ND & 108 & 575 & 604 & $5 / 5$ \\
\hline MPT59 & 1.5 & 2.0 & 24 & 25 & 1.1 & 2.1 & 31 & $4 / 7$ \\
\hline MPT64 & 2.4 & 1.4 & 5.7 & 5.4 & 1.1 & ND & ND & $3 / 5$ \\
\hline MTB38 & 0.9 & 0.7 & 5.8 & 1.1 & 5.2 & ND & ND & $2 / 5$ \\
\hline \multicolumn{9}{|l|}{ Cytosolic antigen } \\
\hline MTB10 & 2.4 & 0.8 & 1.9 & 1.0 & 1.8 & ND & ND & $1 / 5$ \\
\hline MTB70 & 0.7 & 1.3 & 0.8 & 0.7 & 0.5 & ND & ND & $0 / 5$ \\
\hline \multicolumn{9}{|l|}{ Control antigen } \\
\hline MBP & 2.0 & 0.9 & ND & ND & 0.6 & 1.8 & 1.0 & $0 / 5$ \\
\hline
\end{tabular}

$\mathrm{P} / \mathrm{T}=$ Number of patients tested/number of patients positive; $\mathrm{ND}=$ not determined.

The positive responses are $\mathrm{SI}>2.0$.

fined as follows: $\mathrm{SI}=\mathrm{cpm}$ in antigen-stimulated cultures/cpm in cultures without antigen. An SI of $>2$ was considered a positive proliferative response [17]. Percent (\%) positive for a given antigen were calculated from the following formula:

$$
\begin{aligned}
& \% \text { positive }= \\
& \frac{\text { number of patients showing a positive response }}{\text { number of patients tested }} \times 100
\end{aligned}
$$

\section{Results}

The complex antigens of $M$. tuberculosis were strong stimulators of PBMC from TB patients with and without diabetes and healthy subjects, i.e. positive responses were obtained in antigen-induced proliferation assays to all the complex antigens in $>70 \%$ donors (tables $1-3$, respectively). Among the single antigens, the secreted antigens ESAT6, MPT59 and MPT64 were frequently recognized by PBMC of both diabetic and non-diabetic TB patients (tables 1, 2, respectively). However, the best responses were obtained with the M. tuberculosis-specific low-molecular $(6 \mathrm{kDa})$ antigen ESAT6 [positive responders: 5 (71\%) in diabetic and $8(80 \%)$ in non-diabetic TB patients]. All of the single mycobacterial antigens of cytosolic ori- gin were weak stimulators of PBMC ( $\leq 33 \%$ responders) both from diabetic (table 1) and non-diabetic TB patients (table 2). In contrast, the single cytosolic antigens, in particular ML28, ML36 and ML65, were the best stimulators (80, 90 and $70 \%$ responders, respectively) of PBMC from healthy subjects. Among the secreted antigens, the best responses in healthy subjects were seen with the crossreactive antigen MPT59 (80\% responders), whereas ESAT6 was among the weakest stimulators as only $1 / 10$ subjects responded to this antigen (table 3 ).

To identify the antigenic fractions responsible for cellular responses induced by the secreted antigens of M. tuberculosis by diabetic TB patients, PBMC isolated from the blood of 2 diabetic TB patients were stimulated for proliferation in response to the MT-CF fractions F1F10. PBMC from both the patients responded strongly to MT-CF (SI = 632 and 726, table 4). Although both the patients also responded to single secreted antigens ESAT6 and MPT59, the responses to ESAT6 were relatively stronger $(\mathrm{SI}=74$ and 152$)$ than those to MPT59 (SI = 2.1 and 31, table 4). When PBMC from the same patients were tested with MT-CF fractions, all the fractions were stimulatory for the cells from both the patients, confirming the results obtained with single secreted antigens of vary- 
Table 2. Antigen-induced proliferation responses of PBMC from non-diabetic pulmonary TB patients in response to complex and single secreted and cytosolic antigens of $M$. tuberculosis

\begin{tabular}{|c|c|c|c|c|c|c|c|c|c|c|c|}
\hline Antigen & \multicolumn{10}{|c|}{ Antigen-induced proliferation (SI) of PBMC from patients } & $\mathrm{P} / \mathrm{T}$ \\
\hline M. tuberculosis & 94 & 6.0 & 3.7 & 4.0 & 5.5 & 34 & 49 & 4.2 & 1.2 & 12 & $9 / 10$ \\
\hline MT-CF & 62 & 6.2 & 3.2 & 2.6 & 3.1 & 31 & 187 & 199 & 4.5 & 3.0 & $10 / 10$ \\
\hline MT-CW & 160 & 8.2 & 6.6 & 8.8 & 6.5 & 73 & 309 & 425 & 56 & 37 & $10 / 10$ \\
\hline MPT59 & 20 & 0.6 & 55 & 107 & 12 & 3.1 & 63 & 12 & 1.6 & 1.1 & $7 / 10$ \\
\hline MPT64 & 19 & 0.5 & 3.4 & 1.6 & 5.5 & 1.8 & 30 & 5.7 & 1.1 & 1.0 & $5 / 10$ \\
\hline MTB38 & 1.4 & 0.9 & 1.3 & 0.9 & 0.8 & 1.6 & 13 & 1.0 & 1.2 & 1.1 & $1 / 10$ \\
\hline \multicolumn{12}{|l|}{ Cytosolic antigen } \\
\hline MTB10 & 1.4 & 0.6 & 1.6 & 1.9 & 2.0 & 3.1 & 16 & 0.9 & 1.5 & 0.9 & $2 / 10$ \\
\hline MTB70 & 1.4 & 0.5 & 0.4 & 0.4 & 0.9 & 0.7 & 1.9 & 1.1 & 1.1 & 1.0 & $0 / 10$ \\
\hline \multicolumn{12}{|l|}{ Control antigen } \\
\hline MBP & 1.6 & 0.4 & 0.9 & 0.6 & 0.7 & 1.6 & 1.3 & 1.5 & 1.3 & 1.5 & $0 / 10$ \\
\hline
\end{tabular}

$\mathrm{P} / \mathrm{T}=$ Number of patients tested/number of patients positive; $\mathrm{ND}=$ not determined.

The positive responses are $\mathrm{SI}>2.0$.

ing sizes reported above. However, the fractions 1-3 containing low-molecular-weight proteins, including ESAT6, induced strong responses (SI >280) in both the patients (table 4). However, none of the patients responded to the control antigen MBP (table 4). Similar results have previously been reported with PBMC from non-diabetic TB patients when tested with fractionated secreted antigens of $M$. tuberculosis [13].

\section{Discussion}

The worldwide control of TB has two major problems. The first is the non-availability of reagents for specific immunodiagnosis of TB and the second is the lack of a vaccine that can provide consistent protection in all parts of the world [18]. The normally used immunological test for diagnosis of TB is the tuberculin test that uses purified protein derivative of $M$. tuberculosis as a skin test antigen. However, purified protein derivative, being a crude mixture of M. tuberculosis antigens, is not a specific reagent for the diagnosis of TB because it contains antigens that cross-react with environmental mycobacteria and the currently used BCG vaccine [18]. Thus, there is a need to identify $M$. tuberculosis antigens of diagnostic efficacy.

During the past several years, a large number of studies based on CMI responses have been performed to identify mycobacterial antigens of diagnostic potential [18$22]$. These studies have shown that secreted antigens of M. tuberculosis are the primary targets of cellular immune responses in TB patients. However, a study to identify antigens important for recognition by diabetic TB patients in cellular immune responses has been lacking. In the past, a number of assay systems have been used to evaluate CMI responses of PBMC from humans to mycobacterial antigens, which include quantitation of antigeninduced proliferation and secretion of cytokines, e.g. interferon- $\gamma$ and interleukin- 2 by PBMC. Since these tests have been shown to provide similar results in previous studies $[13,14,16,23]$, we have used antigen-induced proliferation assays to evaluate $\mathrm{CMI}$ responses of diabetic $\mathrm{TB}$ patients in this study.

Our results show that $\mathrm{PBMC}$ from diabetic TB patients responded frequently to complex and single secreted antigens (ESAT6, MPT59 and MPT64) of M. tuberculosis in the in vitro antigen-induced proliferation assays. However, the responses to single cytosolic antigens were either 
Table 3. Antigen-induced proliferation responses of PBMC from healthy subjects in response to complex and single secreted and cytosolic antigens of M. tuberculosis

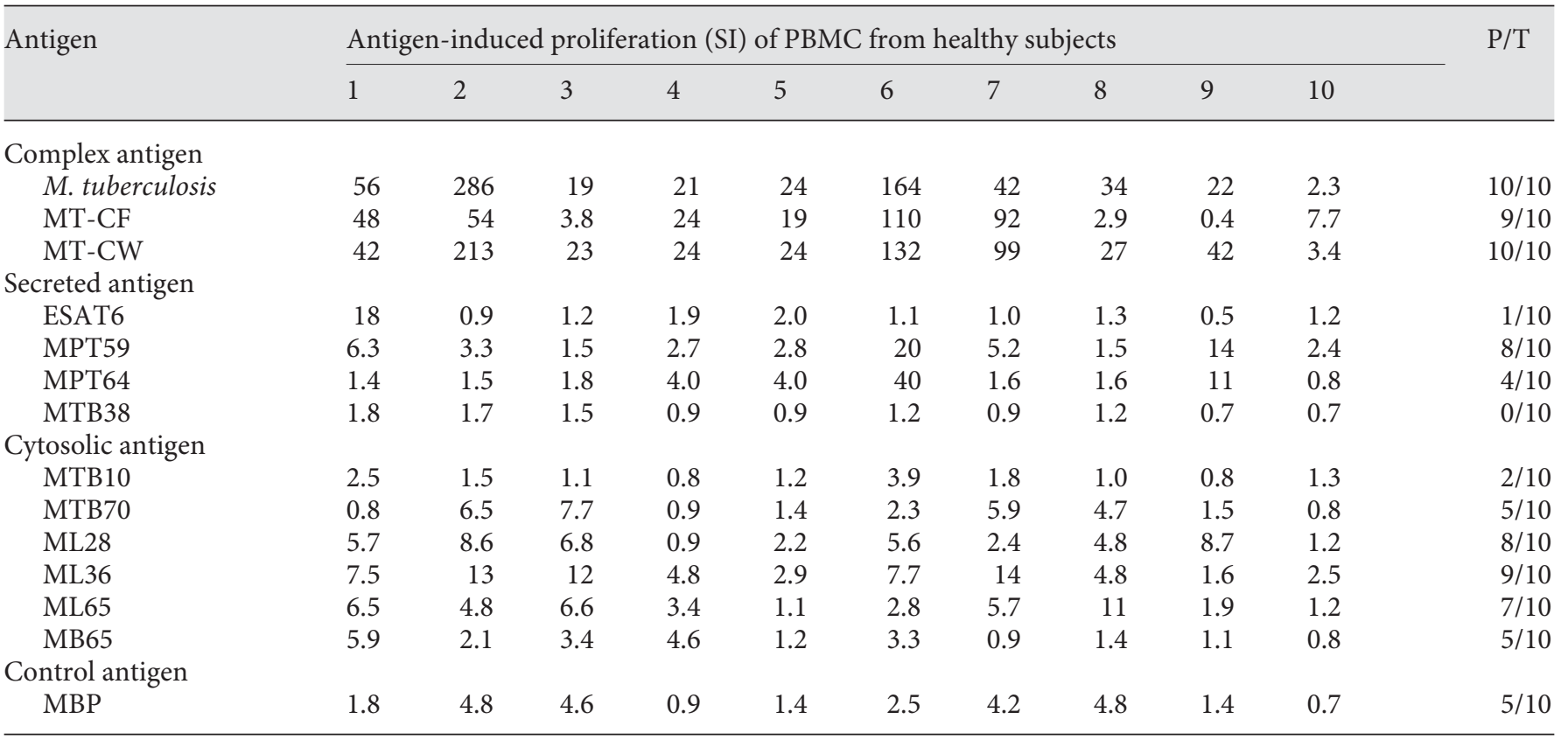

$\mathrm{P} / \mathrm{T}=$ Number of patients tested/number of patients positive; $\mathrm{ND}=$ not determined.

The positive responses are $\mathrm{SI}>2.0$.

not detected or were very weak. Similar results were obtained with PBMC from non-diabetic TB patients included in the study, thus confirming the previous reports [13, $14,16]$. However, in healthy subjects the best responses were obtained with some of the cytosolic mycobacterial antigens used in the study (ML28, ML36 and ML65) along with the cross-reactive secreted antigen MPT59, but not to the M. tuberculosis-specific secreted antigen ESAT6. These results suggest that ESAT6 may be useful in the diagnosis of diabetic as well as non-diabetic TB patients.

Among the single secreted antigens frequently recognized by PBMC of diabetic and non-diabetic TB patients in antigen-induced proliferation assays were antigens of varying size ranging from low- to high-molecular-weight proteins. This was evident from the use of both fractionated (F1-F10) as well as single secreted antigens ESAT6 (6 kDa), MPT59 (35 kDa) and MPT64 (25 kDa). However, among these antigens, only ESAT6 is specific for $M$. $t u$ berculosis as its gene is absent in the BCG vaccine and most environmental mycobacteria, whereas the genes encoding MPT59 and MPT64 are present in BCG as well $[19,24]$. Thus, for diagnostic applications in diabetic as well as non-diabetic TB patients, only ESAT6 and related low molecular weight proteins, e.g. CFP10 that makes
Table 4. Antigen-induced proliferation of PBMC from diabetic pulmonary TB patients in response to MT-CF, ESAT6, MPT59 and fractionated (F1-F10) culture-filtrate antigens of $M$. tuberculosis and MBP

\begin{tabular}{lcc}
\hline $\begin{array}{l}\text { Antigen/CF } \\
\text { fraction }\end{array}$ & \multicolumn{2}{c}{$\begin{array}{c}\text { Antigen-induced proliferation (SI) of } \\
\text { PBMC from diabetic TB patients }\end{array}$} \\
\cline { 2 - 3 } & patient 6 & patient 7 \\
\hline MT-CF & 632 & 726 \\
ESAT6 & 74 & 152 \\
MPT59 & 2.1 & 31 \\
F1 & 342 & 311 \\
F2 & 536 & 624 \\
F3 & 281 & 522 \\
F4 & 67 & 507 \\
F5 & 93 & 532 \\
F6 & 21 & 605 \\
F7 & 13 & 424 \\
F8 & 25 & 365 \\
F9 & 20 & 129 \\
F10 & 142 & 411 \\
MBP & 1.8 & 1.0 \\
\hline
\end{tabular}

The positive responses are $\mathrm{SI}>2.0$. 
1:1 complex with ESAT6 and is encoded by a gene present in the same $M$. tuberculosis-specific region as ESAT6 [25], may be useful. However, since this study is limited with the number of patients, a larger study with these antigens will be required to confirm their potential in the diagnosis of diabetic TB patients using appropriate controls, i.e. non-diabetic TB patients, diabetic patients without TB and healthy subjects without TB and diabetes.

\section{Conclusions}

Diabetic and non-diabetic TB patients respond best to complex and secreted single antigens (ESAT6, MPT59 and MPT64) of M. tuberculosis in cellular immune re- sponses as indicated by antigen-induced proliferation of PBMC. However, only ESAT6 may be useful in the diagnosis of TB in both diabetic and non-diabetic patients because the complex and other secreted single antigens are cross-reactive and induce positive responses in healthy subjects as well.

\section{Acknowledgement}

The work was supported by the Research Administration, Kuwait University, grant No. MI02/02.

\section{References}

1 Borgdorff MW, Floyd K, Broekmans JF: Interventions to reduce tuberculosis mortality and transmission in low- and middle-income countries. Bull World Health Organ 2002;80: 217-227.

22 Dye C: Global epidemiology of tuberculosis. Lancet 2006;36:938-940.

-3 Boutayeb A: The double burden of communicable and non-communicable diseases in developing countries. Trans R Soc Trop Med Hyg 2006;100:191-199.

4 Boutayeb A, Boutayeb S: The burden of non communicable diseases in developing countries. Int J Equity Health 2005;4:2.

5 Broxmeyer L: Diabetes mellitus, tuberculosis and the mycobacteria: two millenia of enigma. Med Hypotheses 2005;65:433-439.

6 Mustafa AS: Development of improved vaccines against tuberculosis. Med Princ Pract 1997;6:55.

7 Mustafa AS, Oftung F: Identification of mycobacterial recombinant antigens recognized by human T cells. Med Princ Pract 1997;6:57-65.

8 Mustafa AS: Development of new vaccines and diagnostic reagents against tuberculosis. Mol Immunol 2002;39:113-119.

-9 Mustafa AS: Mycobacterial gene cloning and expression, comparative genomics, bioinformatics and proteomics in relation to the development of new vaccines and diagnostic reagents. Med Princ Pract 2005;14(suppl 1):2734.

10 Mustafa AS: Recombinant and synthetic peptides to identify Mycobacterium tuberculosis antigens and epitopes of diagnostic and vaccine relevance. Tuberculosis (Edinb) 2005;85: 367-376.

-11 Al-Attiyah R, Shaban FA, Wiker HG, Oftung F, Mustafa AS: Synthetic peptides identify promiscuous human Th1 cell epitopes of the secreted mycobacterial antigen MPB70. Infect Immun 2003;71:1953-1960.
12 Amoudy HA, AI-Asmer ABH, Abul AT, Mustafa AS: Evaluation of complex and defined antigens of Mycobacterium tuberculosis in an IgG specific ELISA for the diagnosis of tuberculosis. Med Princ Pract 1997;6:103-109.

13 Al-Attiyah R, Mustafa AS, Abal AT, Madi NM, Andersen P: Restoration of mycobacterial antigen-induced proliferation and interferon- $\gamma$ responses in peripheral blood mononuclear cells of tuberculosis patients upon effective chemotherapy. FEMS Immunol Med Microbiol 2003;38:249-256.

14 Mustafa AS, Amoudy HA, Oftung F, Wiker HG, Abal AT, Ravn P, Andersen P: Comparison of antigen-specific T-cell responses of tuberculosis patients using complex or single antigens of Mycobacterium tuberculosis. Scand J Immunol 1998;48:535-543.

15 Al-Attiyah R, Mustafa AS: Computer-assisted prediction of HLA-DR binding and experimental analysis for human promiscuous Th1 cell peptides in a novel $24-\mathrm{kDa}$ secreted lipoprotein (LppX) of Mycobacterium tuberculosis. Scand J Immunol 2004;59:16-24.

-16 Al-Attiyah R, Mustafa AS, Abal AT, El-Shamy AM, Dalemans W, Skeiky YAW: In vitro cellular immune responses to complex and newly defined recombinant antigens of Mycobacterium tuberculosis. Clin Exp Immunol 2004; 138:139-144.

17 Mustafa AS, Abal AT, Shaban F, El-Shamy AM, Amoudy HA: HLA-DR binding prediction and experimental evaluation of mycolyl transferase (Ag85B), a major secreted antigen of Mycobacterium tuberculosis. Med Princ Pract 2005;14:140-146.

18 Mustafa AS: Biotechnology in the development of new vaccines and diagnostic reagents against tuberculosis. Curr Pharm Biotechnol 2001;2:157-173.
19 Mustafa AS: Progress towards the development of new anti-tuberculosis vaccines; in Smithe LT (ed): Focus on Tuberculosis Research. New York, Nova Science Publishers, 2005, pp 47-76.

20 Lalvani A: Diagnosing tuberculosis infection in the 21st century: new tools to tackle an old enemy. Chest 2007;131:1898-1906.

- 21 Brodin P, Rosenkrands I, Andersen P, Cole ST, Brosch R: ESAT- 6 proteins: protective antigens and virulence factors? Trends Microbiol 2004;12:500-508.

22 Mantegani P, Piana F, Codecasa L, Galli L, Scarpellini P, Lazzarin A, Cirillo D, Fortis C: Comparison of an in-house and a commercial RD1-based ELISPOT-IFN-gamma assay for the diagnosis of Mycobacterium tuberculosis infection. Clin Med Res 2006;4:266-272.

23 Shinde SR, Chiplunkar SV, Butlin R, Samson PD, Deo MG, Gangal SG: Lymphocyte proliferation, IFN-gamma production and limiting dilution analysis of T-cell responses to ICRC and Mycobacterium leprae antigens in leprosy patients. Int J Lepr Other Mycobact Dis 1993; 61:51-58.

24 Mustafa AS, Al-Attiyah R: Mycobacterium tuberculosis antigens and peptides as new vaccine candidates and immunodiagnostic reagents against tuberculosis. Kuw Med J 2004; 36:171-176.

25 Renshaw PS, Panagiotidou P, Whelan A, Gordon SV, Hewinson RG, Williamson RA, Carr MD: Conclusive evidence that the major Tcell antigens of the Mycobacterium tuberculosis complex ESAT-6 and CFP-10 form a tight, 1:1 complex and characterization of the structural properties of ESAT- 6 , CFP-10, and the ESAT- $6{ }^{*}$ CFP-10 complex: implications for pathogenesis and virulence. J Biol Chem 2002; 277:21598-21603. 